\title{
Characterization of MtoD from Sideroxydans lithotrophicus: a cytochrome $c$ electron shuttle used in lithoautotrophic growth
}

\section{OPEN ACCESS}

Edited by:

Amelia-Elena Rotaru,

University of Southern Denmark,

Denmark

Reviewed by:

Christiane Dahl,

Rheinische

Friedrich-Wilhelms-Universität Bonn,

Germany

Derek R. Lovley,

University of Massachusetts, USA

*Correspondence:

Thomas A. Clarke,

School of Biological Sciences,

University of East Anglia,

University Plain, Norwich NR4 7TJ, UK

tom.clarke@uea.ac.uk

Specialty section:

This article was submitted to Microbial Physiology and Metabolism,

a section of the journal

Frontiers in Microbiology

Received: 03 February 2015

Accepted: 02 April 2015

Published: 28 April 2015

Citation:

Beckwith CR, Edwards MJ, Lawes M, Shi L, Butt JN, Richardson DJ and Clarke TA (2015) Characterization of MtoD from Sideroxydans lithotrophicus: a cytochrome $c$ electron shuttle used in lithoautotrophic growth. Front. Microbiol. 6:332.

doi: 10.3389/fmicb.2015.00332

\begin{abstract}
Christopher R. Beckwith ${ }^{1}$, Marcus J. Edwards' ${ }^{\text {, Matthew Lawes }}{ }^{1}$, Liang Shi ${ }^{2}$, Julea N. Butt ${ }^{1}$, David J. Richardson ${ }^{1}$ and Thomas A. Clarke ${ }^{1 *}$

${ }^{1}$ Centre for Molecular and Structural Biochemistry, School of Biological Sciences and School of Chemistry, University of East Anglia, Norwich, UK, ${ }^{2}$ Pacific Northwest National Laboratory, Richland, WA, USA
\end{abstract}

The autotrophic Sideroxydans lithotrophicus ES-1 can grow by coupling the oxidation of ferrous iron to the reduction of oxygen. Soluble ferrous iron is oxidized at the surface of the cell by an MtoAB porin-cytochrome complex that functions as an electron conduit through the outer membrane. Electrons are then transported to the cytoplasmic membrane where they are used to generate proton motive force (PMF) (for ATP synthesis) and $\mathrm{NADH}$ for autotrophic processes such as carbon fixation. As part of the mtoAB gene cluster, $S$. lithotrophicus also contains the gene $m t o D$ that is proposed to encode a cytochrome c protein. We isolated $m t o D$ from a Shewanella oneidensis expression system where the $m t o D$ gene was expressed on a pBAD plasmid vector. Biochemical, biophysical, and crystallographic characterization of the purified MtoD revealed it as an $11 \mathrm{kDa}$ monomeric protein containing a single heme. Sequence and structural alignment indicated that MtoD belonged to the class- 1 cytochrome $\mathrm{c}$ family and had a similar fold to ferricytochrome c552 family, however the MtoD heme is bis-histidine coordinated and is substantially more exposed than the hemes of other family members. The reduction potential of the MtoD heme at $\mathrm{pH} 7$ was $+155 \mathrm{mV}$ vs. Standard Hydrogen Electrode, which is approximately $100 \mathrm{mV}$ lower than that of mitochondrial cytochrome c. Consideration of the properties of MtoD in the context of the potential respiratory partners identified from the genome suggests that MtoD could associate to multiple electron transfer partners as the primary periplasmic electron shuttle.

Keywords: class 1 cytochrome, MtoD, Sideroxydans lithotrophicus ES-1, iron oxidation

\section{Introduction}

The potential for bacteria to utilize iron as an energy source has been widely recognized in recent years (Bird et al., 2011; Konhauser et al., 2011). Several families of bacteria live at the microoxic/ferrous iron interface where they can survive by coupling the oxidation of ferrous iron to the reduction of oxygen (Hedrich et al., 2011). These bacteria are typically autotrophic and generate sufficient energy from this reaction to fix carbon dioxide and dinitrogen. Bacteria that are capable of this chemolithoautotrophic growth include acidophilic bacteria involved in acid mine drainage such as Acidothiobacillus ferrooxidans and Leptospirillum ferrooxidans 
(Rawlings et al., 1999; Ferguson and Ingledew, 2008; Mishra and Rhee, 2014); the marine stalk-forming Gallionella ferruginea and the freshwater Gallionella capsiferriformans ES-2 and Sideroxydans lithotrophicus ES-1 (Hallbeck et al., 1993; Emerson and Moyer, 1997). The genomes of these organisms have been sequenced revealing a range of putative metabolic pathways (Emerson et al., 2013) and analysis of these pathways poses a range of interesting questions: how do the bacteria extract the electrons from the ferrous iron, and how are those electrons ultimately coupled to the generation of $\mathrm{NADH}$ and a proton motive force (PMF)?

The best studied of the iron oxidizing bacteria is the acidophilic $A$. ferrooxidans, where an outer membrane monoheme Cyc2 collects electrons from the oxidation of $\mathrm{Fe}(\mathrm{II})$ to $\mathrm{Fe}$ (III) and transfers them to a periplasmic rusticyanin which then transfers the electrons to two potential shuttles, the diheme cytochromes Cyc1 and CycA1. These diheme cytochromes then transfer the electrons down divergent routes, either to a cytochrome bcl complex where electrons enter the quinol pool to ultimately generate $\mathrm{NADH}$, or an $\mathrm{aa}_{3}$ oxidase where oxygen is reduced to water together with the transport of protons across the membrane (Bonnefoy and Holmes, 2012; Roger et al., 2012).

The neutrophilic $S$. lithotrophicus ES-1 does not contain any genes with significant homology for $c y c 2$, but instead contains the genes mtoA (Slit_2497) and $m t o B$ (Slit_2496) (Liu et al., 2012). These are homologs of Shewanella oneidensis $m \operatorname{tr} A$ and $m \operatorname{tr} B$, which encode a decaheme cytochrome MtrA and transmembrane porin MtrB. These two proteins form a porin-cytochrome complex in the outer membrane of $S$. oneidensis that allows for efficient electron transport through the outer membrane via a chain of hemes (Hartshorne et al., 2009; White et al., 2013). It has been proposed that MtoA and MtoB fulfill a similar function in S. lithotrophicus ES-1, in forming an electron conduit that allows electrons to be collected from the oxidation of ferrous iron at the surface of the cell and transported through to the periplasm (Liu et al., 2012).

Both $m t o A$ and $m t o B$ are located in a gene cluster in the S. lithotrophicus ES-1 genome that also contains two other $c$ type cytochromes; Slit_2498 that encodes MtoD, a small monoheme cytochrome and Slit_2495, which encodes CymAES-1, a tetraheme quinol oxidoreductase. As part of the same cluster it is possible that all four of these genes are co-transcribed and expressed as part of an operon. This would provide a porincytochrome complex (MtoAB), a soluble periplasmic cytochrome (MtoD), and a quinol oxidoreductase in the cytoplasmic membrane (CymAES-1) (Emerson et al., 2013).

S. lithotrophicus ES-1 also contains the genes necessary to express two distinct oxygenases, a cytochrome $b_{3}$ oxidase $\left(\mathrm{cbb}_{3}\right)$, and a cytochrome $\mathrm{bd}_{1}$ oxidase $\left(\mathrm{cbd}_{1}\right)$. These have been characterized in other bacteria and shown to have low $K_{M}$-values for oxygen: typically $\mathrm{cbd}_{1}$ operates in the micromolar range while $\mathrm{cbb}_{3}$ operates in the sub-micromolar range. $\mathrm{cbb}_{3}$ couples $\mathrm{O}_{2}$ reduction to proton pumping and receives electrons from a cytochrome $c$, while $\mathrm{cbd}_{1}$ has no proton pumping mechanism and receives electrons directly from the quinol pool (Pitcher and Watmough, 2004).
The bioenergetic and biochemical mechanism of precisely how iron oxidizing bacteria are able to couple $\mathrm{Fe}$ oxidation to $\mathrm{O}_{2}$ reduction is still unclear. How do electrons from the MtoAB complex enter the cytochrome oxidase? It was previously suggested that $\mathrm{CymA}_{E S-1}$ was the redox partner, however, this would result in the loss of protonmotive force. In order to better understand the possible roles of the periplasmic redox partners in S. lithotrophicus ES-1 the MtoD cytochrome was expressed in a recombinant form, purified and characterized using a range of biochemical, spectroscopic, and crystallographic techniques.

\section{Materials and Methods}

\section{Expression and Isolation of Strep-II Tagged MtoD}

The 354 bp sequence encoding $m t o D$ was synthesized and cloned into a puc57 vector by GENScript. $m$ toD was amplified from pUC57 using the following primers: $m t o D \_1 \_F: 5^{\prime}$-ATG ACT CGT CAA GCT TAT TCC TCA ATG TTG and $m t o D \_1 \_R:$ 5'-GAG CGA AAG GAT CCA GTC CAC CAG. A second pair of primers were used to make modifications to $m t o D$ including the addition of a $5^{\prime}$ CACC overhang, making the $m t o D$ insert compatible with the desired pBAD202 D-TOPO cloning kit, followed by a ribosome binding sequence and a 3' 6xCAC repeat coding for a C-terminal polyhistidine tag. $m t o D \_2 \_F: 5^{\prime}$-CAC CTA AGA AGG AGA TAT ACA TCC CAT GAC TCG TCA AGC TTA TTC. $m t o D \_R \_6 x H i s: 5^{\prime}$ CTA GTG GTG GTG GTG GTG GTG GAG CGA AAG GAT C. A pBAD directional TOPO ${ }^{\circledR}$ expression kit was used clone $m t o D$-His into a pBAD202 expression vector. One Shot TOP10 E. coli cells were transformed with pBAD202_mtoDHis using methods described in the pBAD D-TOPO user guide. pBAD202_mtoD-His was conjugated from TOP10 to $S$. oneidensis MR-1 using E. coli helper strain DH5 $\alpha$ pRK2013. Kanamycin and carbenicillin were used to select for successfully conjugated MR-1. DNA sequencing performed by Eurofins MWG operon using primers $m t o D \_2 \_F$ and $m t o D \_\mathrm{R} \_6 \mathrm{xHis}$ confirmed successful conjugation of pBAD202_mtoD-His into $S$. oneidensis MR-1. For the production of Strep II-tagged MtoD pBAD202_mtoD-His was isolated from S. oneidensis MR-1 using a miniprep kit and the whole plasmid was amplified using the primers $m$ toD_SII_F: $5^{\prime}$-AAT TCG AGA AGT AGA AGG GCG AGC TCA AGC TTG AAG GTA and mtoD_SII_R: 5' GTG GAT GAG ACC AGA GCG AAA GGA TCC AGT CCA CAG G. The pBAD202_mtoD-His template was removed by DpnI digestion followed by PCR clean up. T4 polynucleotide kinase was used to phosphorylate the linear product and a blunt end ligation was performed using DNA ligase to circularize the pBAD202_mtoD-SII product. One Shot TOP10 E. coli were transformed with pBAD202_mtoD-SII and conjugation was used to produce recombinant $S$. oneidensis MR-1 as before.

Recombinant $S$. oneidensis MR-1_mtoD-SII was cultured aerobically at $30^{\circ} \mathrm{C}$ in $20 \mathrm{~L}$ batches in LB media. Expression of tagged protein was induced at mid-exponential phase $\left(\mathrm{OD}_{600}\right.$ : $0.5)$ by addition of L-arabinose to a working concentration of $2 \mathrm{mM}$. Cultures were incubated for a further $5 \mathrm{~h}$ and harvested by centrifugation at $6000 \mathrm{~g}$ for $15 \mathrm{~min}$. Recombinant MR-1 cell pellets were re-suspended in $20 \mathrm{mM}$ HEPES pH 7 buffer and 
three passes of French Press treatment at 1000 psi $(6.89 \mathrm{MPa})$ were used to lyse the cells. The soluble cell fraction was isolated by ultracentrifugation of the lysate at $205,000 \mathrm{~g}$ for $2 \mathrm{~h}$. The supernatant was retained for purification of tagged MtoD. MtoD was isolated using $5 \mathrm{~mL}$ Strep-Tactin affinity column (GE healthcare). The column was run with a $20 \mathrm{mM}$ HEPES $\mathrm{pH} 7,150 \mathrm{mM} \mathrm{NaCl}$ equilibration/wash buffer. Four cycles of loading and eluting were performed to isolate all of the expressed MtoD. SDS PAGE was used to analyze eluted fractions; MtoD fractions were dialysed with $20 \mathrm{mM}$ HEPES pH 7, $150 \mathrm{mM} \mathrm{NaCl}$ overnight then concentrated in preparation for size exclusion chromatography. Gel filtration was performed using a Superdex S75 16/60 column. The column was equilibrated with $20 \mathrm{mM}$ HEPES pH 7, $150 \mathrm{mM} \mathrm{NaCl}$ before loading MtoD and running at a flow rate of $0.5 \mathrm{~mL} \cdot \mathrm{min}^{-1}$. Pure fractions of MtoD, determined by SDS PAGE analysis, were pooled, concentrated and dialysed with $20 \mathrm{mM}$ HEPES pH 7, $100 \mathrm{mM} \mathrm{NaCl}$ overnight at $4^{\circ} \mathrm{C}$.

\section{Pyridine Hemochrome Analysis of MtoD}

Oxidized spectra of horse heart cytochrome $\mathrm{c}$ and MtoD were prepared in $20 \mathrm{mM}$ HEPES $\mathrm{pH} 7$ buffer containing $2 \mathrm{mM}$ $\mathrm{CaCl}_{2}$ for cytochrome $\mathrm{c}$, and $100 \mathrm{mM} \mathrm{NaCl}$ for MtoD. Each sample was fully oxidized with $10 \mu \mathrm{M} \mathrm{K} \mathrm{K}_{3} \mathrm{Fe}(\mathrm{CN})_{6}$ before measurement of UV/vis electronic absorption spectrum. The pyridine hemochrome method was used to quantify the concentration of heme in the purified MtoD sample and to determine the extinction coefficient of the Soret maxima of MtoD (Berry and Trumpower, 1987). Briefly cytochrome $\mathrm{c}$ and MtoD were mixed with pyridine and $\mathrm{NaOH}$ to give final concentrations of 3 and $75 \mathrm{mM}$, respectively. These samples were divided into two Suba-sealed quartz cuvettes. Samples were oxidized using $10 \mu \mathrm{M} \mathrm{K} \mathrm{K}_{3} \mathrm{Fe}(\mathrm{CN})_{6}$ and reduced with $3 \mathrm{mM} \mathrm{Na} 2 \mathrm{~S}_{2} \mathrm{O}_{4}$ to give the oxidized and reduced spectra of heme bis-pyridine. A BioRad protein assay kit was used to determine the total protein concentration.

\section{Sedimentation Velocity}

Four hundred and ten microliters of MtoD samples of 3.5 and $8.5 \mu \mathrm{M}$ were measured into sample chambers of a two chamber

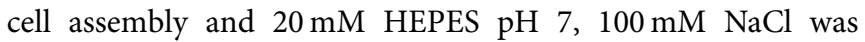
measured into the reference chambers. A Beckman Optima XLA analytical ultracentrifuge with an An50Ti rotor was used to optically measure sedimentation, monitoring absorbance at $406 \mathrm{~nm}$ with a rotational speed of $42,000 \mathrm{rpm}$. 300 radial scans were performed over a 20 -h period at a constant temperature of $20^{\circ} \mathrm{C}$. Analyses of the sedimentation velocity data were performed using SEDFIT (Brown and Schuck, 2006). The C(s) distribution model was applied to the data and non-linear fitting was performed. Buffer density and viscosity parameters (1.0039 g.mL $\mathrm{mL}^{-1}$ and $1.0264 \times 10^{-2} \rho$, respectively) in addition to predicted v-bar for MtoD $\left(0.7282 \mathrm{~mL} . \mathrm{g}^{-1}\right)$, were included in the $\mathrm{C}(\mathrm{s})$ model to solve Lamm equations.

\section{MtoD Crystallization and Data Collection}

A pure solution of MtoD was concentrated to $30 \mathrm{mg} \cdot \mathrm{mL}^{-1}$ and sparse matrix screening, using the sitting drop method, was performed to explore potential crystallization conditions.
Crystals formed at $16^{\circ} \mathrm{C}$ in $0.6 \mu \mathrm{l}$ drops at ratios of $1: 1$ or $2: 1$ mother liquor/protein. The mother liquor was $0.1 \mathrm{M} \mathrm{KCN}, 30 \%$ PEG 2000 MME. Crystals were cryo-protected by transferring to a solution of mother liquor containing $12 \%$ glycerol before being vitrified by plunging into liquid nitrogen. Data were collected on MtoD crystals in a gaseous stream of nitrogen at $100 \mathrm{~K}$ on beamlines I03 at the Diamond Light Source (UK). MtoD crystals were of space group P22 ${ }_{1} 2_{1}$ with typical cell dimensions of $a=29.70, b=40.20, c=92.180 \AA$ A. A SAD dataset was collected at a wavelength of $1.72 \AA$ to a final resolution of $2.5 \AA$. Further datasets from single crystals were collected using an $\mathrm{x}$-ray wavelength of $0.97 \AA$.

\section{MtoD Structure Determination and Refinement}

MtoD datasets were processed using XIA2 (Winter, 2010). The SAD dataset of MtoD was analyzed using the autosol pipeline within the PHENIX software suite (Adams et al., 2010). The program HySS located one heavy atom site and the electron density maps calculated with PHASER/RESOLVE were sufficiently interpretable to manually place a single heme corresponding to a single MtoD molecule in the asymmetric unit. The model building program Phenix AutoBuild was used to build residues followed by alternating rounds of manual building and refinement using PHENIX (Adams et al., 2010) or REFMAC (Murshudov et al., 2011). The final model was refined to an Rcryst (Rfree) value of 19.4 (24.5)\%. This model has no residues in the disallowed region of the Ramachadran plot. Coordinates have been deposited in the RCSB Protein Data bank under accession code 4XXL.

\section{Electrochemistry}

Mediated redox spectropotentiometry of a solution of MtoD was performed using methods described previously (Bamford et al., 2002). Experiments were performed under continuous argon gas flow with a solution of $5.5 \mu \mathrm{M}$ MtoD in $20 \mathrm{mM}$

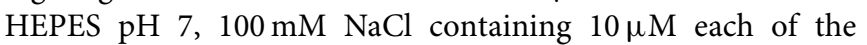
following mediators: 2,3,5,6-tetramethyl-p-phenylenediamine (DAD), phenazine methosulphate (PMS), phenazine ethosulphate (PES), 5-hydroxy-1,4-napthoquinone, 2,3,5,6tetramethyl-1,4-benzoquinone, 2-methyl-1,4-napthoquinone, 9,10-anthraquinone-2,6-disulphonic acid, anthraquinone-2sulphonic acid and $1,1^{\prime}$-dibenzyl-4,4' -bipyridinium dichloride. The electrochemical potential was raised or lowered through the addition of aliquots of anaerobic solutions containing potassium ferricyanide or sodium dithionite, respectively.

\section{Results}

\section{Expression, Purification, and Characterization of S. lithotrophicus MtoD}

MtoD was purified to homogeneity from $S$. oneidensis as described in Methods. SDS-PAGE analysis revealed that MtoD ran as a single band with apparent molecular weight of $13 \mathrm{kDa}$, slightly larger than the predicted molecular weight of $11 \mathrm{kDa}$ due to the c-terminal strep-II tag (Figure 1A). Edman degradation (PNAC facility, Cambridge UK) revealed the $\mathrm{N}$ terminal sequence began AVDVD, matching the cleavage site 


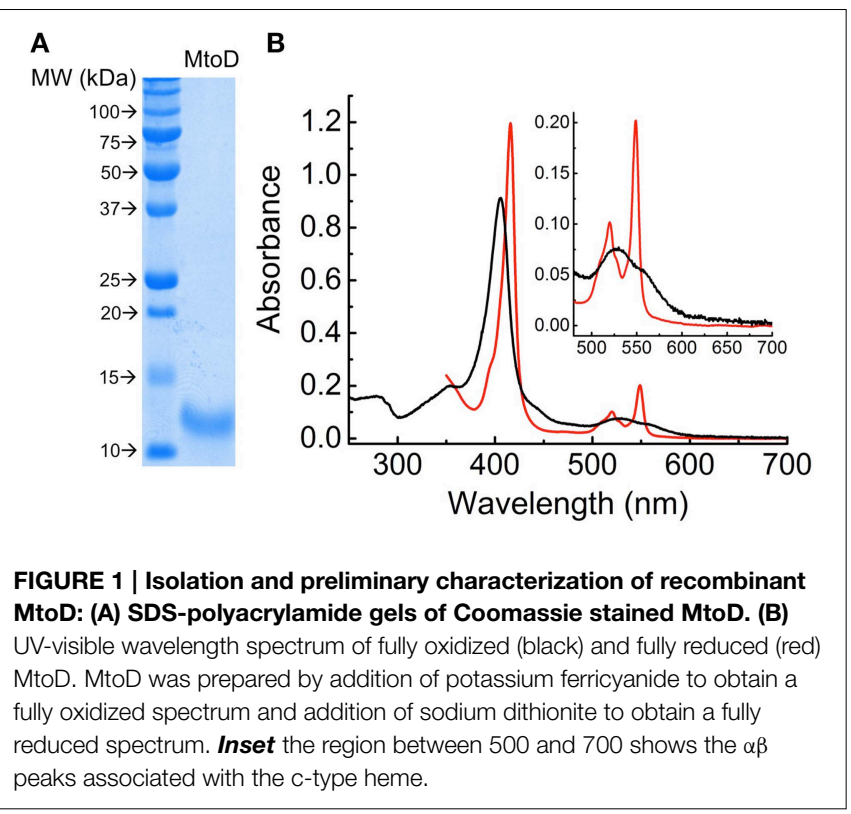

predicted by SignalP (Petersen et al., 2011) and pyridine hemochrome assays revealed that the sample of MtoD contained approximately stoichiometric ratio of 0.85 heme: protein, giving an $\varepsilon_{410}$ coefficient of $105.2 \mathrm{mM}^{-1} \mathrm{~cm}^{-1}$.

Solutions of MtoD display electronic absorbance spectra consistent with the presence of a low-spin c-type heme (Figure 1B). The characteristic Soret peak in the oxidized protein has a maximum at $406 \mathrm{~nm}$, giving a $280 / 406 \mathrm{~nm}$ absorbance ratio of 0.19 . The $406 \mathrm{~nm}$ maximum shifts to $416 \mathrm{~nm}$ on reduction of MtoD and $\alpha$ and $\beta$ peaks appear with maxima at 549 and $520 \mathrm{~nm}$, respectively. Spectral features above $600 \mathrm{~nm}$ would be indicative of high-spin ferric heme or low-spin ferric heme with His/Met axial heme ligation as is typically observed in other class1 cytochrome-c family members (Bertini et al., 2006). However, MtoD displayed no detectable absorbance above $600 \mathrm{~nm}$ in agreement with the heme ligand set resolved by X-ray diffraction as described below. Mediated potentiometric titration of MtoD monitored by electronic absorbance spectroscopy defined the heme redox properties (Figure $\mathbf{2 A}$ ). Changes in the absorbance at $549 \mathrm{~nm}$ due to the ferric/ferrous heme interconversion were fully reversible with change of solution potential (Figure 2B). The behavior was in excellent agreement with that predicted by the Nernst equation for a single redox center undergoing a one-electron redox transformation with a mid-point potential of $155 \pm 10 \mathrm{mV}$ vs. SHE.

The biophysical properties of MtoD in solution were examined using sedimentation velocity. MtoD samples at 3.5 and $8.5 \mu \mathrm{M}$ were centrifuged as described in methods and the migration profile of MtoD at $406 \mathrm{~nm}$ was measured over $5 \mathrm{~h}$ (Figure 3A). The data was fitted using the software program SEDFIT which revealed a single species with a sedimentation coefficient of $1.55 \mathrm{~s}$ and a molecular mass of $11.7 \mathrm{kDa}$ (Figure 3B). Further analysis of the sedimentation data gave a $\mathrm{f} / \mathrm{f}_{o}$ coefficient of 1.24 , indicating that MtoD behaved like a monomeric globular protein in solution.
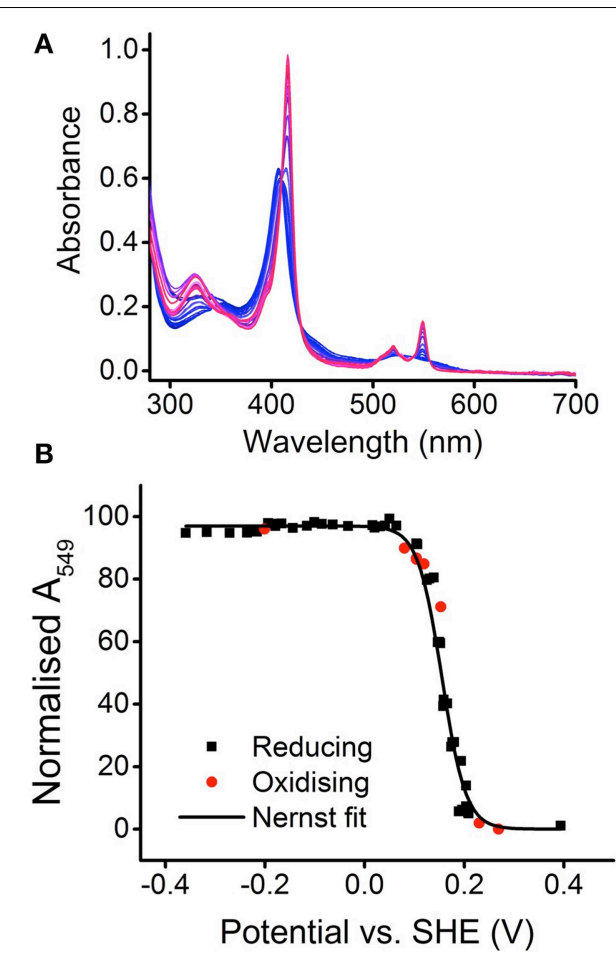

FIGURE 2 | Redox characterization of MtoD. (A) Electronic absorbance of MtoD equilibrated at potentials between 0.4 and $-0.4 \mathrm{~V}$ during a mediated potentiometric titration. Spectra are colored from blue to red corresponding to increasingly reduced protein. (B) Variation of absorbance at $549 \mathrm{~nm}\left(\mathrm{~A}_{549}\right)$ with solution potential (points) and best fit (line) to the Nernst equation for a one-electron transformation with a mid-point potential of $+155 \mathrm{mV}$ vs. SHE. Experiments performed in $20 \mathrm{mM}$ HEPES $\mathrm{pH} 7,100 \mathrm{mM} \mathrm{NaCl}$ at $21^{\circ} \mathrm{C}$ with a mediator range as listed in the methods.

The crystal structure of MtoD was solved to an initial resolution of $2.29 \AA$ by single wavelength anomalous dispersion (SAD) using the anomalous signal caused by the single iron atom contained within the heme group. The initial model was used as a template for molecular replacement to obtain a final resolution of $1.47 \AA$. The overall statistics obtained for data collection and structure refinement are given in Supplementary Table 1 . The crystal structure contained residues 28-119 of the processed amino acid sequence and a single c-type heme covalently attached to $\mathrm{Cys}_{43}$ and $\mathrm{Cys}_{46}$ via thioether bonds (Figure 4A), consistent with the histidine ligands predicted from the sequence alignment. The iron atom of the heme group was coordinated by the imidazoles of the porin cofactor and $\mathrm{His}_{47}$ and His95 of the active site. The imidazole side chains of the two histidines coordinating the heme iron are arranged near-parallel relative to one another, at an angle of approximately $35^{\circ}$ (Figure 4B).

The sequence alignment between $\mathrm{MtoD}$ and the other structurally resolved cytochromes revealed that cytochrome c552 from Nitrosomas europea had the highest amino acid sequence homology (Supplemental Figure 1). When the structure of MtoD was compared with the other available structures of bacterial monoheme c-type cytochromes, the closest structural homolog was cytochrome c552 from Hydrogenobacter thermophilus (PDB 


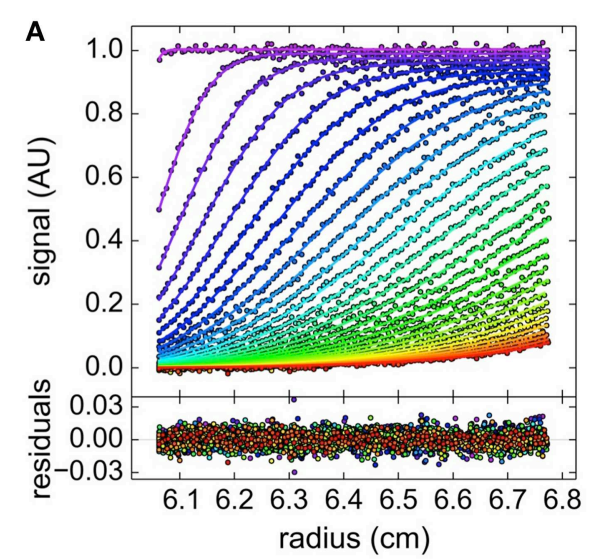

B

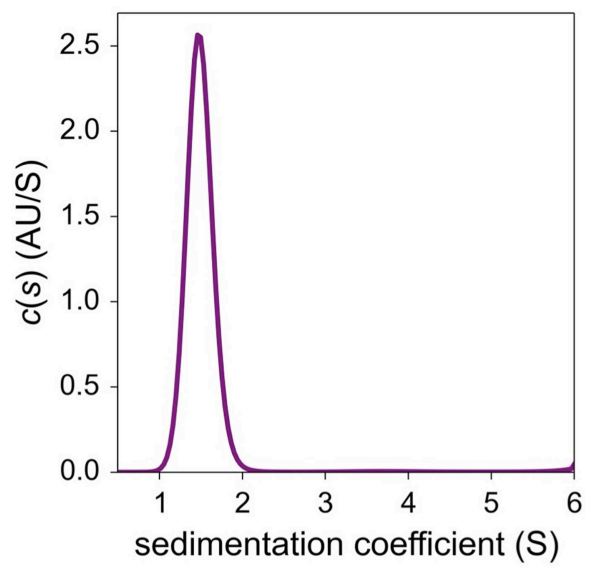

FIGURE 3 | Solution based biophysical characterization using sedimentation velocity. (A) Absorption at $406 \mathrm{~nm}$ was used to track the boundary migration of an $8.5 \mu \mathrm{M}$ sample of MtoD in $20 \mathrm{mM}$ HEPES $\mathrm{pH} 7$, $100 \mathrm{mM} \mathrm{NaCl}$ at $42,000 \mathrm{rpm}$. The change in absorbance was fitted using SEDFIT with the residual fit shown below the data (B). The c(s) distribution of the fitted data indicates the presence of a single, non-interacting species with a sedimentation coefficient of $1.55 \mathrm{~s}$ and a corresponding molecular weight of $11.7 \mathrm{kDa}$, corrected for temperature and buffer parameters.

id. 1YNR). Superposition of the main chain of this cytochrome with MtoD gave an average root-mean-square-displacement of $2.59 \AA$ (Figure 4B, Table 1). Despite the high level of secondary structure conservation there are notable structural differences between MtoD and the other known c-type cytochromes. One obvious difference is that the axial coordination of the MtoD heme is bis-Histidine, while other crystallized cytochromes have Histidine/Methionine coordination (Table 1). The class 1 cytochromes typically contain a flexible loop that covers the front of the heme, however in MtoD this region is restrained by a hydrogen bonding network that causes the formation of a $\beta$-loop and prevents the peptide sidechains from interacting with the heme propionate groups (Figures 4C,D). This causes an increase in the overall surface exposure of the MtoD heme, giving an overall exposed heme of $152 \AA^{2}$ compared to 30-61 $\AA^{2}$ for other class 1 cytochromes. Typically the hemes of these class 1 cytochromes are exposed on one side, next to the thiolated cysteine residues, with the propionate edge being completely covered. To date the heme group of MtoD is significantly more
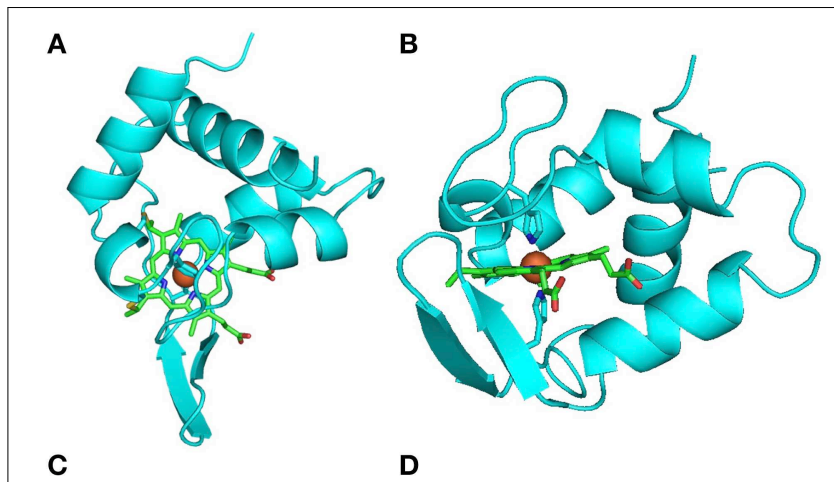

C

D
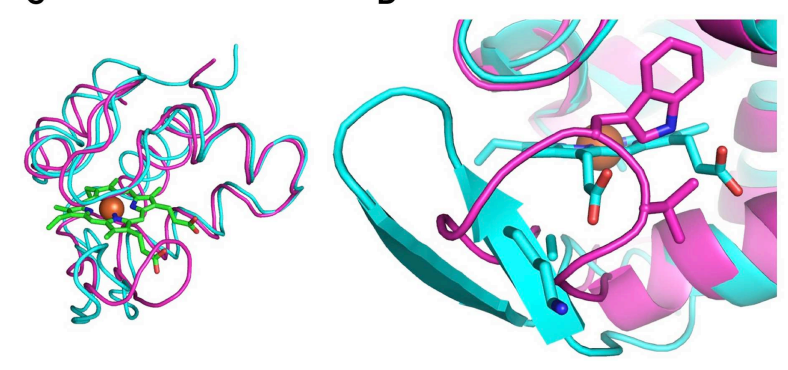

FIGURE 4 | 1.47Å Crystal structure of MtoD. (A) Top view of MtoD; (B) Side view of MtoD revealing the near-parallel histidine side chains co-ordinating the heme iron. (C) Superposition of MtoD (cyan) over the secondary structure of cytochrome c552 from Hydrogenbacter thermophilus (magenta) (D) Close up of the loop region that exposes the MtoD heme. In cytochrome c552 the conserved tryptophan Trp54 covers much of the exposed heme.

TABLE 1 | Structural comparison of MtoD with similar monoheme c-type cytochromes.

\begin{tabular}{|c|c|c|c|c|c|}
\hline Name & Class & PDB id & $\begin{array}{c}\text { Axial } \\
\text { ligands }\end{array}$ & $\begin{array}{c}\text { Accesible } \\
\text { Heme } \\
\text { area }\left(\AA^{2}\right)\end{array}$ & $\begin{array}{c}\text { RMSD } \\
\text { (Å) }\end{array}$ \\
\hline S. lithotrophicus MtoD & - & $4 X X L$ & His/His & 152.5 & - \\
\hline N. europea c552 & c552 & $1 \mathrm{~A} 56$ & His/Met & 54.2 & 3.23 \\
\hline P. aeruginosa c551 & c551 & $451 \mathrm{C}$ & His/Met & 52.6 & 3.25 \\
\hline H. thermoluteolus c552 & c552 & 2DOS & His/Met & 61.3 & 2.87 \\
\hline H. thermophilus c552 & c552 & 1YNR & His/Met & 57.4 & 2.59 \\
\hline P. stutzeri c551 & c552 & $1 \mathrm{COR}$ & His/Met & 55.2 & 3.08 \\
\hline A. aeolicus c555 & c555 & $2 Z X Y$ & His/Met & 37.5 & 3.54 \\
\hline
\end{tabular}

Six cytochromes were identified through sequence similarity to MtoD using BLAST. Structures were downloaded from the RCSB protein data bank. The root mean square displacement (RMSD) was measured using SUPERPOSE (Krissinel and Henrick, 2004). Accesible heme area was measured using a $1.4 \AA$ probe using AREAIMOL (Shrake and Rupley, 1973).

exposed to solvent than the heme of any other structurally resolved monoheme cytochrome, suggesting that the properties of the heme are likely to be extremely susceptible to changes within the local environment.

\section{Discussion}

Autotrophic iron oxidizing bacteria face a significant bioenergetic challenge in generating both the reducing equivalents $(\mathrm{NADH})$ and chemical energy (ATP) required 
for carbon fixation and other anabolic reactions essential for cell survival. The energy source for both NADH and ATP production is generated from the liberation of electrons obtained from the oxidation of iron at the cell surface. At $\mathrm{pH} 7.0$ the redox potential of this iron couple $\mathrm{Fe}(\mathrm{OH})_{3} / \mathrm{Fe}^{2+}$ is $-236 \mathrm{mV}$ vs. SHE, which is low enough to allow electron transfer across the outer membrane through the MtoAB complex (Widdel et al., 1993; Liu et al., 2012). The electrons obtained from iron oxidation are required both for PMF-coupled oxygen reduction, and the reduction of $\mathrm{NADH}$ at the cytoplasmic membrane. At an initial potential around $-236 \mathrm{mV}$ the energy associated with these electrons would be sufficient to catalyze the reduction of oxygen $(+816 \mathrm{mV})$, but they would not be able to reduce $\mathrm{NAD}^{+}$to $\mathrm{NADH}(-320 \mathrm{mV})$. As a consequence electrons destined for $\mathrm{NADH}$ formation will have to be pumped "uphill" through different carriers before reducing NADH. The energy source for this uphill electron transfer is most likely to be obtained from the proton gradient. In A. ferrooxidans two periplasmic diheme cytochromes $\mathrm{Cycl}$ and CycAl take electrons to either the cytochrome oxidase or the cytochrome $\mathrm{bc}_{1}$ complex, which then runs in reverse to reduce ubiquinone. These cytochromes are located in two separate operons; the rus operon contains $c y c 1$ as well as $c y c 2, \operatorname{rus} A$ and the genes for a cytochrome $\mathrm{c}$ oxidase, while the petl operon contains $c y c A 1$ and the genes for a cytochrome $\mathrm{bc}_{1}$ complex (Roger et al., 2012). In contrast the mto gene cluster of $S$. lithotrophicus contains the gene for only one monoheme cytochrome: $m t o D$, while the gene clusters that contain the genes of cytochrome $b_{1}$ and cytochrome $b_{3}$ do not contain any soluble monoheme cytochromes.
The MtoD cytochrome has a number of unusual features. Comparative analysis of the MtoD amino acid sequence with the sequences of other structurally defined class- 1 cytochromes revealed that MtoD is unusual in having bis-His coordination instead of His/Met, and that the sequence around the distal ligand is not proline rich as observed for other cytochromes (Table 1 and Supplemental Figure 1). The measured redox potential of $+155 \mathrm{mV}$ for the MtoD heme is high for a bis-His coordinated heme and the heme group is significantly more solvent exposed than in monoheme cytochromes. It is possible that these unusual features of the MtoD heme group allow it to function as an electron donor to cytochrome $b_{3}$, cytochrome $b_{1}$ and/or $\mathrm{CymA}_{\mathrm{ES}-1}$ (Figure 5).

Previous studies on the potentials of the heme groups of MtoA indicate that they span a mid-point potential range between +30 and $-350 \mathrm{mV}$ (Liu et al., 2012), which is lower than the potential of MtoD and indicates that electron transfer from MtoA to MtoD would be thermodynamically favorable. This supports the hypothesis that MtoD could be the electron transfer shuttle between the outer membrane MtoAB complex and the cytochrome $\mathrm{bb}_{3}$ oxidase on the cytoplasmic membrane, allowing the efficient generation of a PMF.

A membrane bound $\mathrm{NADH}$ dehydrogenase is proposed to reduce $\mathrm{NAD}^{+}$to NADH using electrons provided by the quinol pool and energy generated by the PMF (Emerson et al., 2013). Either the cytochrome bcl complex or CymA $A_{E S-1}$ could generate quinol, using electrons supplied by MtoD (Figure 5). At $+155 \mathrm{mV}$, the potential of MtoD is only slightly higher than the midpoint potential of ubiquinol $(+100 \mathrm{mV}$ vs. SHE)

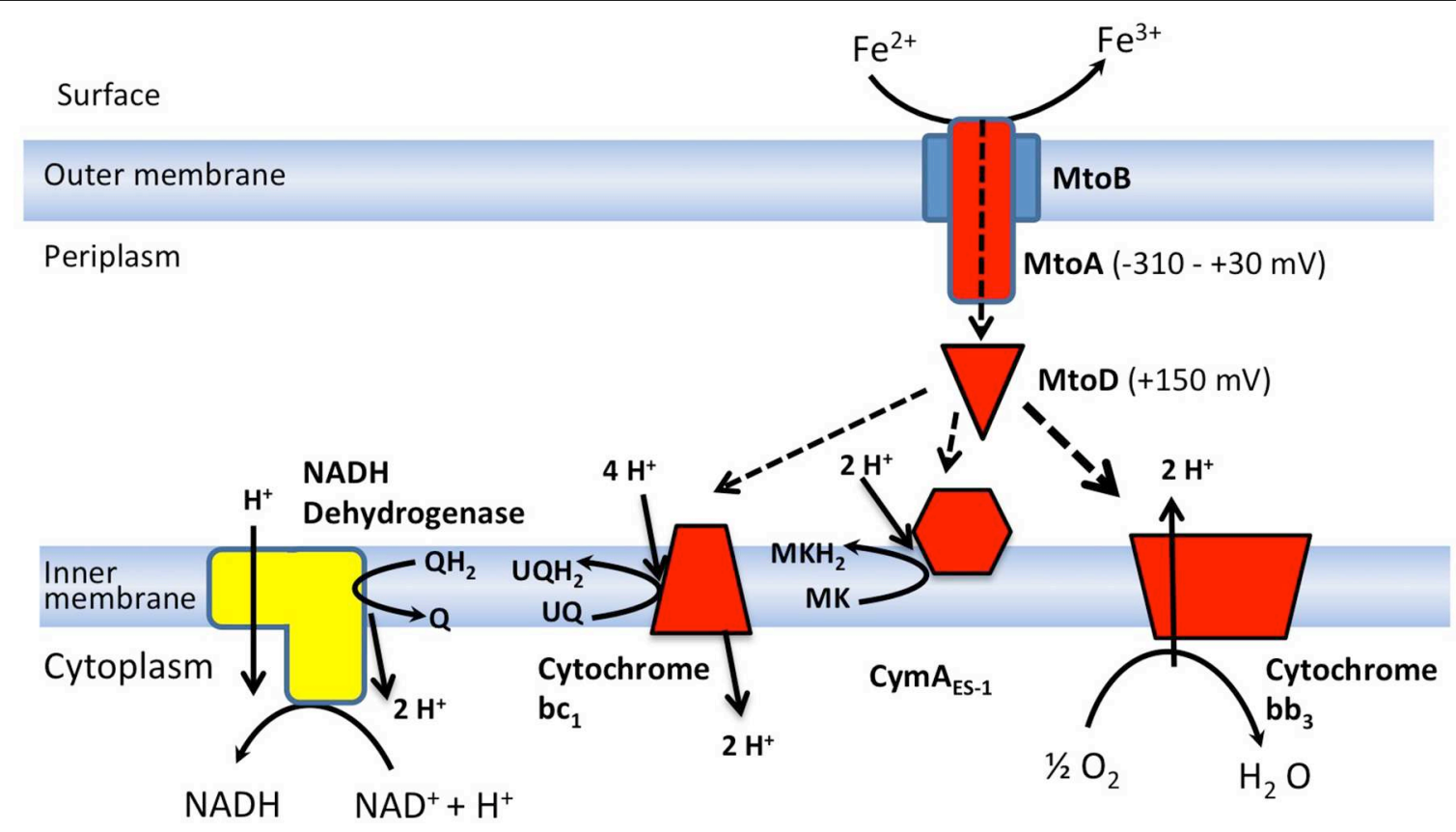

FIGURE 5 | Proposed dual role of MtoD in supporting both proton translocation and NADH production. Possible electron transfer pathways through MtoA and MtoD are indicated by dashed lines. $U Q$ and $U_{2} H_{2}$ refer to ubiquinone and ubiquinol respectively, while $\mathrm{MK}$ and $\mathrm{MKH}_{2}$ refer to menaquinone and menaquinol. $\mathrm{Q}$ and $\mathrm{QH}_{2}$ represent non-specific quinones and quinols. The known redox midpoint ponentials for S. lithotrophicus ES-1 MtoD and MtoA are shown in parentheses. 
and consequently electron transfer from MtoD to cytochrome $\mathrm{bc}_{1}$ could occur in order to drive NADH production. The generation of reduced ubiquinol from cytochrome bc1 has been observed in other iron oxidizing bacteria, notably A. ferrooxidans (Elbehti et al., 2000). In this system, the two half reactions of the cytochrome bcl complex run in reverse, causing the net transport of two protons across the cytoplasmic membrane for every ubiquinone reduced.

The role of CymA $A_{E S-1}$ is less clear. The homologous CymA cytochrome from $S$. oneidensis MR-1 was shown to be specific for menaquinone, that has a midpoint potential of $-70 \mathrm{mV}$ vs. SHE (McMillan et al., 2012). This suggests that the measured potential of MtoD would not allow thermodynamically favorable electron transfer to CymA $\mathrm{ES}_{\mathrm{E}-1}$. However, the highly exposed heme surface of MtoD is likely to be sensitive to changes in the local environment, such as those caused by protein complex formation. This may allow lowering of the MtoD midpoint potential on association with $\mathrm{Cym} \mathrm{A}_{\mathrm{ES}-1}$, facilitating electron exchange and menaquinone reduction.

In summary, the structure and electrochemical properties of MtoD are consistent with its possible role as an electron shuttle between MtoAB on the outer membrane and a range of potential electron acceptors on the inner membrane. However, a full biochemical analysis will be required to verify the true redox partners and confirm the pathway of electron transfer through this iron oxidizing bacterium.

\section{References}

Adams, P. D., Afonine, P. V., Bunkoczi, G., Chen, V. B., Davis, I. W., Echols, N., et al. (2010). PHENIX: a comprehensive Python-based system for macromolecular structure solution. Acta Crystallogr. D Biol. Crystallogr. 66, 213-221. doi: 10.1107/S0907444909052925

Bamford, V. A., Angove, H. C., Seward, H. E., Thomson, A. J., Cole, J. A., Butt, J. N., et al. (2002). Structure and spectroscopy of the periplasmic cytochrome c nitrite reductase from Escherichia coli. Biochemistry 41, 2921-2931. doi: 10.1021/bi015765d

Berry, E. A., and Trumpower, B. L. (1987). Simultaneous determination of Hemesa, Hemes-B, and Hemes-C from pyridine hemochrome spectra. Anal. Biochem. 161, 1-15. doi: 10.1016/0003-2697(87)90643-9

Bertini, I., Cavallaro, G., and Rosato, A. (2006). Cytochrome c: occurrence and functions. Chem. Rev. 106, 90-115. doi: 10.1021/cr050241v

Bird, L. J., Bonnefoy, V., and Newman, D. K. (2011). Bioenergetic challenges of microbial iron metabolisms. Trends Microbiol. 19, 330-340. doi: 10.1016/j.tim.2011.05.001

Bonnefoy, V., and Holmes, D. S. (2012). Genomic insights into microbial iron oxidation and iron uptake strategies in extremely acidic environments. Environ. Microbiol. 14, 1597-1611. doi: 10.1111/j.1462-2920.2011.02626.x

Brown, P. H., and Schuck, P. (2006). Macromolecular size-and-shape distributions by sedimentation velocity analytical ultracentrifugation. Biophys. J. 90, 4651-4661. doi: 10.1529/biophysj.106.081372

Elbehti, A., Brasseur, G., and Lemesle-Meunier, D. (2000). First evidence for existence of an uphill electron transfer through the $\mathrm{bc}(1)$ and NADH$\mathrm{Q}$ oxidoreductase complexes of the acidophilic obligate chemolithotrophic ferrous ion-oxidizing bacterium Thiobacillus ferrooxidans. J. Bacteriol. 182, 3602-3606. doi: 10.1128/JB.182.12.3602-3606.2000

Emerson, D., Field, E. K., Chertkov, O., Davenport, K. W., Goodwin, L., Munk, C., et al. (2013). Comparative genomics of freshwater Fe-oxidizing bacteria: implications for physiology, ecology, and systematics. Front. Microbiol. 4:254. doi: $10.3389 /$ fmicb.2013.00254

\section{Author Contributions}

$\mathrm{CB}, \mathrm{ME}, \mathrm{ML}$, and LS carried out data acquisition and analysis. $\mathrm{CB}, \mathrm{ME}, \mathrm{JB}, \mathrm{DR}, \mathrm{TC}$ carried out data analysis and interpretation. $\mathrm{CB}, \mathrm{ME}, \mathrm{ML}, \mathrm{LS}, \mathrm{JB}, \mathrm{DR}$, TC drafted the manuscript. CB, ME, JB, $\mathrm{DR}, \mathrm{TC}$ revised the manuscript. All authors approved the final manuscript.

\section{Acknowledgments}

This research was supported by the Biotechnology and Biological Sciences Research Council (BB/K009885/1, BB/L023733/1) and a DTP studentship to ML. the U.S. Department of Energy (DOE), Office of Biological and Environmental Research (BER) through the Subsurface Biogeochemical Research (SBR) program, and is a contribution of Pacific Northwest National Laboratory (PNNL) SBR SFA. PNNL is operated for DOE by Battelle under contract DE-AC05-76RLO1830. We are grateful to Prof. Jim Fredrickson and John Zachara for useful discussion.

\section{Supplementary Material}

The Supplementary Material for this article can be found online at: http://journal.frontiersin.org/article/10.3389/fmicb. 2015.00332/abstract

Emerson, D., and Moyer, C. (1997). Isolation and characterization of novel ironoxidizing bacteria that grow at circumneutral pH. Appl. Environ. Microbiol. 63, 4784-4792.

Ferguson, S. J., and Ingledew, W. J. (2008). Energetic problems faced by microorganisms growing or surviving on parsimonious energy sources and at acidic pH: I. Acidithiobacillus ferrooxidans as a paradigm. Biochim. Biophys. Acta Bioenerg. 1777, 1471-1479. doi: 10.3389/fmicb.2012.00037

Hallbeck, L., Stahl, F., and Pedersen, K. (1993). Phylogeny and phenotypic characterization of the stalk-forming and iron-oxidizing bacterium Gallionellaferruginea. J. Gen. Microbiol. 139, 1531-1535. doi: 10.1099/00221287-139-71531

Hartshorne, R. S., Reardon, C. L., Ross, D., Nuester, J., Clarke, T. A., Gates, A. J., et al. (2009). Characterization of an electron conduit between bacteria and the extracellular environment. Proc. Natl. Acad. Sci. U.S.A. 106, 22169-22174. doi: 10.1073/pnas.0900086106

Hedrich, S., Schlomann, M., and Johnson, D. B. (2011). The iron-oxidizing proteobacteria. Microbiology 157, 1551-1564. doi: 10.1099/mic.0.045344-0

Konhauser, K. O., Kappler, A., and Roden, E. E. (2011). Iron in microbial metabolisms. Elements 7, 89-93. doi: 10.2113/gselements.7.2.89

Krissinel, E., and Henrick, K. (2004). Secondary-structure matching (SSM), a new tool for fast protein structure alignment in three dimensions. Acta Crystallogr. D Biol. Crystallogr. 60, 2256-2268. doi: 10.1107/S0907444904026460

Liu, J., Wang, Z. M., Belchik, S. M., Edwards, M. J., Liu, C. X., Kennedy, D. W., et al. (2012). Identification and characterization of MtoA: a decaheme c-type cytochrome of the neutrophilic Fe(II)-oxidizing bacterium Sideroxydans lithotrophicus ES-1. Front. Microbiol. 3:37. doi: 10.3389/fmicb.2012.00037

McMillan, D. G., Marritt, S. J., Butt, J. N., and Jeuken, L. J. (2012). Menaquinone7 is specific cofactor in tetraheme quinol dehydrogenase CymA. J. Biol. Chem. 287, 14215-14225. doi: 10.1074/jbc.M112.348813

Mishra, D., and Rhee, Y. H. (2014). Microbial leaching of metals from solid industrial wastes. J. Microbiol. 52, 1-7. doi: 10.1007/s12275-014-3532-3

Murshudov, G. N., Skubak, P., Lebedev, A. A., Pannu, N. S., Steiner, R. A., Nicholls, R. A., et al. (2011). REFMAC5 for the refinement of macromolecular 
crystal structures. Acta Crystallogr. D Biol. Crystallogr. 67, 355-367. doi: 10.1107/S0907444911001314

Petersen, T. N., Brunak, S., von Heijne, G., and Nielsen, H. (2011). SignalP 4.0: discriminating signal peptides from transmembrane regions. Nat. Methods 8, 785-786. doi: 10.1038/nmeth.1701

Pitcher, R. S., and Watmough, N. J. (2004). The bacterial cytochrome cbb(3) oxidases. Biochim. Biophys. Acta Bioenerg. 1655, 388-399. doi: 10.1016/j.bbabio.2003.09.017

Rawlings, D. E., Tributsch, H., and Hansford, G. S. (1999). Reasons why 'Leptospirillum'-like species rather than Thiobacillus ferrooxidans are the dominant iron-oxidizing bacteria in many commercial processes for the biooxidation of pyrite and related ores. Microbiology 145, 5-13. doi: 10.1099/13500872-145-1-5

Roger, M., Castelle, C., Guiral, M., Infossi, P., Lojou, E., Giudici-Orticoni, M. T., et al. (2012). Mineral respiration under extreme acidic conditions: from a supramolecular organization to a molecular adaptation in Acidithiobacillus ferrooxidans. Biochem. Soc. Trans. 40, 1324-1329. doi: 10.1042/BST201 20141

Shrake, A., and Rupley, J. A. (1973). Environment and exposure to solvent of protein atoms - lysozyme and insulin. J. Mol. Biol. 79, 351-371. doi: 10.1016/0022-2836(73)90011-9
White, G. F., Shi, Z., Shi, L., Wang, Z. M., Dohnalkova, A. C., Marshall, M. J., et al. (2013). Rapid electron exchange between surface-exposed bacterial cytochromes and Fe(III) minerals. Proc. Natl. Acad. Sci. U.S.A. 110, 6346-6351. doi: 10.1073/pnas.1220074110

Widdel, F., Schnell, S., Heising, S., Ehrenreich, A., Assmus, B., and Schink, B. (1993). Ferrous iron oxidation by anoxygenic phototrophic bacteria. Nature 362, 834-836. doi: 10.1038/362834a0

Winter, G. (2010). xia2: an expert system for macromolecular crystallography data reduction. J. Appl. Crystallogr. 43, 186-190. doi: 10.1107/S0021889809045701

Conflict of Interest Statement: The authors declare that the research was conducted in the absence of any commercial or financial relationships that could be construed as a potential conflict of interest.

Copyright (c) 2015 Beckwith, Edwards, Lawes, Shi, Butt, Richardson and Clarke. This is an open-access article distributed under the terms of the Creative Commons Attribution License (CC BY). The use, distribution or reproduction in other forums is permitted, provided the original author(s) or licensor are credited and that the original publication in this journal is cited, in accordance with accepted academic practice. No use, distribution or reproduction is permitted which does not comply with these terms. 